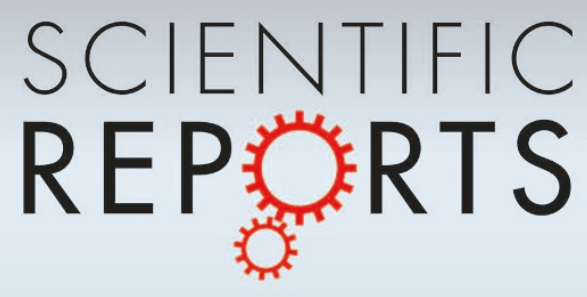

OPEN

SUBJECT AREAS:

BIOPHYSICAL CHEMISTRY

BIOCHEMICAL NETWORKS

COMPUTATIONAL BIOPHYSICS

Received

7 November 2014

Accepted

24 February 2015

Published

15 May 2015

Correspondence and requests for materials should be addressed to A.B. (adriano.barra@ romal.infn.it)

\section{Notes on stochastic (bio)-logic gates: computing with allosteric cooperativity}

\author{
Elena Agliari ${ }^{1}$, Matteo Altavilla ${ }^{2}$, Adriano Barra' ${ }^{1}$ Lorenzo Dello Schiavo ${ }^{2}$ \& Evgeny Katz ${ }^{3}$ \\ ${ }^{1}$ Dipartimento di Fisica, Sapienza Università di Roma, Italy, ${ }^{2}$ Dipartimento di Matematica, Sapienza Università di Roma, Italy,
${ }^{3}$ Department of Chemistry and Biomolecular Science, Clarkson University, New York, USA.
}

Recent experimental breakthroughs have finally allowed to implement in-vitro reaction kinetics (the so called enzyme based logic) which code for two-inputs logic gates and mimic the stochastic AND (and NAND) as well as the stochastic OR (and NOR). This accomplishment, together with the already-known single-input gates (performing as YES and NOT), provides a logic base and paves the way to the development of powerful biotechnological devices. However, as biochemical systems are always affected by the presence of noise (e.g. thermal), standard logic is not the correct theoretical reference framework, rather we show that statistical mechanics can work for this scope: here we formulate a complete statistical mechanical description of the Monod-Wyman-Changeaux allosteric model for both single and double ligand systems, with the purpose of exploring their practical capabilities to express noisy logical operators and/or perform stochastic logical operations. Mixing statistical mechanics with logics, and testing quantitatively the resulting findings on the available biochemical data, we successfully revise the concept of cooperativity (and anti-cooperativity) for allosteric systems, with particular emphasis on its computational capabilities, the related ranges and scaling of the involved parameters and its differences with classical cooperativity (and anti-cooperativity).

C ell's life is based on a hierarchical and modular organization of interactions among its molecules': a functional module is defined as a discrete ensemble of reactions whose functions are separable from those of other molecules. Such a separation can be of spatial origin (processes ruled by short range interactions) or of chemical origin (processes requiring specific interactions) $)^{2}$. The latter, i.e., chemical specificity, is at the basis of biological information processing ${ }^{3,4}$. A paradigmatic example of this is the signal transduction pathway of the so called two signal model in immunology by which an effector lymphocyte needs two signals (both integrated on its membrane's highly-specific receptors in a close temporal interval) to get active $e^{5}$ these signals are the presence of the antigen (via the complex MHC-TCR) and the consensus of an helper-cell (via CD40 and an eliciting cytokine); this constitutes a biological, stochastic AND gate ${ }^{6}$. We added the adjective stochastic because, quoting Germain, "as one dissects the immune system at finer and finer levels of resolution, there is actually a decreasing predictability in the behavior of any particular unit of function", furthermore, "no individual cell requires two signals (1/4) rather, the probability that many cells will divide more often is increased by co-stimulation"': as a result, standard logic (where operations follow a deterministic chain) plays as the ideal reference framework, while an operative one -a stochastic formulation of logic- should take into account the presence of noise too.

Beyond countless natural examples, biologic gates have been realized even experimentally, see e.g. Refs. 8-18, the ultimate goal being the realization of stochastic, yet controllable, biological circuits ${ }^{19-22}$.

Such striking outcomes also arouse a great theoretical attention aimed to develop a self-contained framework able to highlight their potentialities and suggest possible developments. In particular, statistical mechanics has proved to be a proper candidate tool for unveiling biological complexity: in the past two decades statistical mechanics has been applied to investigate intra-cellular (e.g. metabolomics ${ }^{23,24}$, proteinomics ${ }^{25,26}$ ) as well as extra-cellular (e.g. neural networks ${ }^{3,27}$, immune networks ${ }^{28,29}$ ) systems. Also, statistical mechanics intrinsically offers a partially-random scaffold which is the ideal setting for a stochastic logic gate theory.

Another route to unveil the spontaneous information processing capabilities of biological matters is naturally constituted by information theory (see e.g. Refs. 30,31 and references therein): remarkably, statistical mechanics and information theory (see the seminal works by Khinchin ${ }^{32,33}$, and by Jaynes ${ }^{34,35}$ ) and, in turn, information theory and logics (see the seminal works by Von Neumann ${ }^{36}$, and by Chaitin ${ }^{37}$ ) have been highlighted to be deeply connected. Therefore, it is not surprising that even in the quantitative modeling of biological phenomena these two routes are not conflicting but, rather, complementary. 
In this work, we will use the former (statistical mechanics) to describe a huge variety of biochemical allosteric reactions, and then, through the latter (mathematical logic), we will show how these reactions naturally encode stochastic versions of boolean gates and are thus capable of noisy information processing.

We will especially focus on allosteric reactions (as those of Koshland, Nemethy and Filmer (KNF) ${ }^{38}$ and Monod-WymanChangeaux $(\mathrm{MWC})^{39}$ ) as they play a major role in enzymatic processes for which a great amount of experimental data is nowadays available. However, classical reaction kinetics (i.e. those coded by Hill, Adair, etc. ${ }^{40}$ ) can also perform logical calculations and they have been set in a statistical mechanical scaffold in Ref. 19: along the paper we will deepen the crucial differences between the two types of kinetics -allosteric cooperativity versus standard cooperativity- when framed within statistical mechanics.

\section{Results}

In the case of allosteric receptors, several models have been introduced. Many of these assume that a receptor can exist in either an active or inactive state, and that binding of a ligand biases the receptor to one of the two states. In particular, in the Monod-WymanChangeaux (MWC) model, ligand-bound receptors can be in either state, but coupled receptors switch between states in synchrony. Beyond that pioneering work, several models able to provide qualitative and quantitative descriptions of binding phenomena have been further introduced in the Literature, as e.g. the sequential model by Koshland, Nemethy and Filmer (KNF).

Here we consider MWC-like kinetics, and we reformulate it into a statistical mechanical framework. We start by introducing terminology and parameters for mono-receptor/mono-ligand systems (playing for single input gates as YES and NOT) and then we expand such a scenario in order to account for the kinetics of more complex systems (double-receptors/double-ligands, as those will play for two-input gates as AND, NAND, OR, NOR).

The plan is as follows: Once introduced the microscopic settings (e.g., the occupancy states $\sigma_{i}, i \in(1,1 / 4, n)$ of $n$ receptors and the dissociation energy $h$ ), we define the Hamiltonian functions $H(\sigma, h)$ coding for the chemical bindings; then -being $\beta$ the thermal noise $\beta=1 / k_{B} T$ (where $k_{B}$ is the Boltzmann constant and $T$ represents the temperature) - we build the related Maxwell-Boltzmann probabilistic weights $\propto \exp [-\beta H(\sigma, h)]$. The latter allows computing the partition functions $Z=\sum_{\sigma} \exp (-\beta H)$, both for the active state $Z_{A}$ and for the inactive $Z_{I}$ state; the ratios, $p_{A}=Z_{A} /\left(Z_{A}+Z_{I}\right)$ and $p_{I}=Z_{I} /\left(Z_{A}+Z_{I}\right)$ then return the probabilities of the active/inactive states as functions of the parameters (e.g. $\beta, h, n$ ).

These probabilities are first analyzed from a logic perspective in order to show how they can account for boolean gates and then used to successfully fit the outcomes of the experiments on enzyme based logic. This route, although rather lengthy, shows why allosteric mechanisms share similar behaviors with those of classical cooperativity, but, at the same time, clearly reveals deep differences between these phenomena.

System description. Specifically, we start considering a system built of several molecules, each displaying one or more receptors. Each receptor exhibits multiple binding sites where a ligand can reversibly bind, and which can exist in two different states (i.e. active and inactive). In general the receptors exhibited by a given molecule can differ in e.g., the number of binding sites, the affinity with ligands, etc. As we are building a theory for single and double input gates, in the following, we will focus on simple systems where receptors can house only one or two kinds of binding sites, as exemplified in Fig. 1.

The simplest system we consider is made of a set of receptors of the same kind and in the presence of a unique ligand (see panel $a$ in Fig. 1). More precisely, each receptor is constituted by $n$ functionally
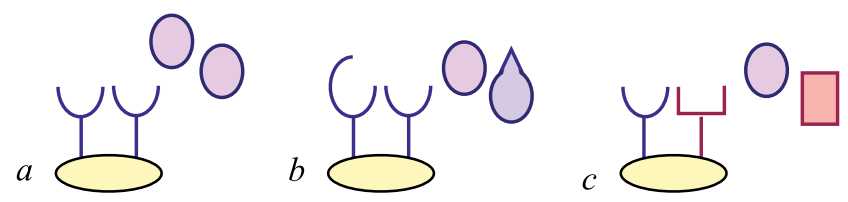

Figure 1 | This scheme summarizes the kind of systems we are considering here: Mono-receptor/Mono-ligand (a), Mono-receptor/Double-ligand ( $b$ ) and Double-receptor/Double-ligand $(c)$. In this cartoon all molecules are shown as dimeric, but cases $a$ and $b$ also work with monomeric structures. In the Mono-receptor/Mono-ligand case only one kind of receptor and one kind of ligand (compatible with the receptor) are considered; in the Monoreceptor/Double-ligand case we still have one kind of receptor, but two different ligands both compatible with the receptor; in the Doublereceptor/Double-ligand case we consider molecules displaying two different receptors in the presence of two different ligands, each compatible with only one receptor. The kinetics of these systems is addressed in System description Section, while in Section Logical Operation they are shown to work as YES, OR, and AND logic gates. See also Ref. 42.

identical binding sites indexed by $i$, whose occupancy is given by a boolean vector $\sigma=\left\{\sigma_{i}\right\}, i=1,1 / 4, n$ where $\sigma_{i}=1$ (respectively 0 ) indicates that the binding site $i$ is occupied (respectively vacant).

As required by the all-or-none MWC model, each receptor is either active (T) or inactive ( $\mathrm{R})$; the receptor state is indicated by a boolean activation parameter $a,(a=0,1)^{41,42}$.

In the absence of the ligand, the active and inactive states (which are assumed to be in equilibrium) differ in their chemical potential, whose delta, indicated by $E$, can, in principle, be either positive (favoring the inactive state) or negative (favoring the active state): note that, the presence of a difference $E$ in energy between the active and inactive states implies an exponentially unbalanced ratio between their relative concentrations (ruled by the MaxwellBoltzmann weight).

Given a system of receptor molecules in the absence of ligand and in equilibrium at a given temperature $T$, we pose the following assumptions:

(a) As both the active and inactive state may coexist, the composition of the system also depends on the parameter $L \equiv L(\beta)>0$, namely the equilibrium constant at inverse temperature $\beta$ (in proper units, namely setting the Boltzmann constant $K_{B} \equiv 1$ ). Letting $[R]$ be the total concentration of the receptors, $\left[R_{A}\right]$ (respectively $\left[R_{I}\right]$ ) the concentration of the active (respectively inactive) receptors in the in absence of ligand, it is $[R]=\left[R_{A}\right]+$ $\left[R_{I}\right]$ and $\left[R_{A}\right]=L\left[R_{I}\right]$

(b) For the sake of simplicity, binding sites of a mono-receptor are considered as functionally identical (as in the original model ${ }^{39}$ ).

In the absence of ligand, we also need to establish which of the two states (namely the active and inactive one) has a higher chemical potential. As shown in the Literature (see Ref. 41 and below) the choice is in general arbitrary (i.e. case dependent), hence we take both possibilities into account. We therefore consider two sets of mutually exclusive assumptions (the latter of which is denoted by a "prime" symbol).

(c) The active state has a higher chemical potential. Notice that, while this assumption is in contrast with the original MWC model $^{39}$, the model itself is still self consistent as thoroughly explained in Ref. 41. The same conclusion may be drawn by the fact that, in the MWC paper, the opposite assumption is merely exploited for calculations. (i.e. $E>0$ ), as e.g. in Refs. 41,43 , hence the inactive state must then be predominant (to minimize energy) (i.e. $L \ll 1$ ); 
$\left(c^{\prime}\right)$ The active state has a lower chemical potential (i.e. $E^{\prime} \equiv-E<$ 0 ) as e.g. in the original MWC model ${ }^{39}$, hence (still for minimum energy requirement) the active state must then be predominant (i.e. $L \gg 1$ ).

For a thorough comparison of these two alternative assumptions (and those of the original MWC) we refer to Tab. 1.

For the sake of clarity we will from now on refer to the (c)-type assumptions as "assumptions $\mathcal{A}$ " and to the $\left(c^{\prime}\right)$-type assumptions as

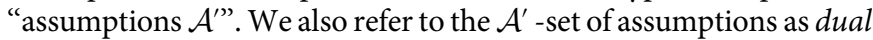
to assumptions $\mathcal{A}$, where this terminology is introduced to match the one of mathematical logic and will be therefore explained in Section "Logical operations". All the assumptions without a dual one are taken to be part of both the assumption sets.

Let us now discuss the case of a system of receptor molecules in the presence of ligand. Clearly, the behavior of the system is expected to depend on ligand's concentration $[S]$ and on the receptor state (i.e. either active or inactive). The dependence on the receptor state is formalized by introducing dissociation constants $K_{A}$ and $K_{I}$ for the receptor in the active and inactive state, respectively (see Ref. 42). Letting $\left[\left(R_{A} S\right)_{i}\right]$ be the concentration of the receptor/ligand complex's molecules which have exactly $i$ occupied binding sites, we define the average concentration of the active receptor/ligand complex as

$$
\left\langle\left[R_{A} S\right]\right\rangle \equiv \frac{1}{n} \sum_{i=1}^{n} i \cdot\left[\left(R_{A} S\right)_{i}\right]
$$

We can define the average concentration $\left\langle\left[R_{I} S\right]\right\rangle$ of the inactive receptor/ligand complex in an analogous way, and we can then set

$$
K_{A} \equiv \frac{[S][R]}{\left\langle\left[R_{A} S\right]\right\rangle}, \quad K_{I} \equiv \frac{[S][R]}{\left\langle\left[R_{I} S\right]\right\rangle},
$$

in accordance with the original presentation of MWC model (in Ref. 39 , p. 90, microscopic dissociation constants of a ligand [1/4] bound to a stereospecific site are considered, whose arithmetic weighted means we denote as global dissociation constants.). The dynamics of the receptor/ligand system is therefore determined by the variable $[S]$ and the parameters $K_{A}, K_{I}$.

Now, considering both the ligand and the receptor/ligand solution we assume that

(d) receptor-ligand solution is homogeneous and isotropic. This mean-field-like assumption is actually a key assumption of all the approaches in modeling classical reaction kinetics, see e.g. Ref. 19.

Finally, a ligand can play as an activator (i.e. its presence enhances the receptor activation) as well as a suppressor (i.e. its presence hindrances receptor's activation) depending on the chemical potential time by time associated to the chemical reaction under examination (assumptions (c)'s). This choice is dual and will be deepened later: to avoid trivial (i.e. static) behavior of the system, we have to set either

(e) The ligand is an activator, i.e. the presence of the ligand enhances activation of the receptor. Therefore, the occupation of each receptor singularly decreases the energy required for activation by a parameter $\epsilon>0$.

\section{AUT}

$\left(e^{\prime}\right)$ The ligand is a suppressor, i.e. the presence of the ligand hindrances activation of the receptor. Therefore, the occupation of each receptor singularly increases the energy required for activation by a parameter $\epsilon>0$.

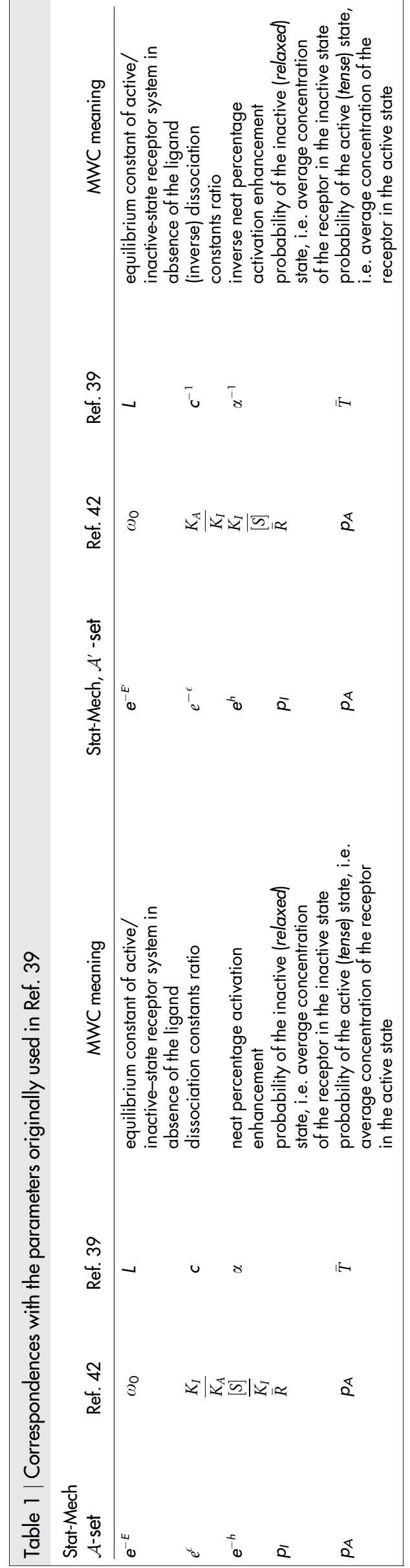


Weight

State

Energy Stat. Mech. Chem.

$a=0$
$\sigma=0$

0

$1 / Z$

$1 / Z$

$a=1$
$\sigma=0$

E

$e^{-E} / Z$

$L / Z$

$a=0$
$\sigma=1$

$h$

$e^{-h} / Z$

$\alpha / Z$

$a=1$
$\sigma=1$

$E-\varepsilon+h \quad e^{-(E-\epsilon+h)} / Z \quad \alpha c L / Z$

Figure $2 \mid$ This scheme summarizes the states and the weights of the simplest MWC molecule (that, in turn, codes for the YES gate). Having only one binding site $(n=1)$, the number of possible states is four, from top to bottom: inactive and vacant, active and vacant, inactive and occupied, active and occupied. Each state corresponds to an energy, to a statistical mechanics weight and to a chemical weight. The energy is obtained by considering both the conformational degree of freedom of the molecule and the free energy of the binding process. The statistical mechanics weight is obtained according to the Boltzmann factor and the chemical weight is derived according to Tab. 1. See also Ref. 48.

Mono receptor/Mono ligand (MM) properties at equilibrium. Under assumptions $\mathcal{A}$, any mono-receptor/mono-ligand system, built by $n$ receptors $[i \in(1,1 / 4, n)]$, and whose occupancy is ruled by $\sigma_{i}=(0,1)$, can be described by the following allosteric Hamiltonian function

$$
H(\sigma, a)=\left(E-\epsilon \sum_{i=1}^{n} \sigma_{i}\right) a+h \sum_{i=1}^{n} \sigma_{i},
$$

where we recall that $E$ is the energy delta given by chemical potential, and $h$ is the dissociation energy, namely the energy captured by a single binding site of the inactive state receptor by binding to a ligand molecule; the term in the brackets accounts for the fact that ligand acts as an activator since, for the active state $(a=1)$ binding is energetically favored, while in the inactive state $(a=0)$ the related term disappears in the Hamiltonian that reduces to the last term accounting for the bare association energy only.

By the same reasoning under assumptions $\mathcal{A}^{\prime}$, we obtain

$$
H(\sigma, a)=\left(-E+\epsilon \sum_{i=1}^{n} \sigma_{i}\right) a+h \sum_{i=1}^{n} \sigma_{i},
$$

The main features of the mono-receptor/mono-ligand systems described above are summarized in Fig. 2.

It is worth highlighting that the Hamiltonians (3) and (4) do not include any two-body couplings, i.e. any term $\propto \sum_{i j} \sigma_{i} \sigma_{j}$ : this framework is intrinsically one-body in the statistical mechanical vocabulary and this has implications in biochemistry too. For instance, one-body models do not undergo phase transitions and, as the latter mirror ultrasensitive reactions in chemical kinetics ${ }^{19}$, those are ruled out by these Hamiltonians.

Since the activation parameter is boolean, the receptor/ligand complex state may be considered regardless of the state of the receptor, by introducing the two Hamiltonians $H_{A}(\sigma) \equiv H(\sigma, 1)$ and $H_{I}(\sigma) \equiv H(\sigma, 0)$, defining the active and the inactive state energy, respectively. The corresponding partition functions are

$$
\begin{aligned}
& Z_{A}=\sum_{\{\sigma\}} e^{-\beta H_{A}(\sigma)} \\
& Z_{I}=\sum_{\{\sigma\}} e^{-\beta H_{I}(\sigma)},
\end{aligned}
$$

while the total partition function $Z$ is given by

$$
Z=\sum_{\{\sigma\},\{a\}} e^{-\beta H(\sigma, a)}=Z_{A}+Z_{I} .
$$

A few remarks are in order here:

- The summations in the partition function (7) account for the activation degree of freedom too. This means that the latter participate in thermalization or, in other words, that the intrinsic timescale for the dynamics of $a$ is bounded from above by those of the $\sigma$ : this is consistent with the original MWC assumptions of synchronized switches among coupled receptors (the so called all-or-none behavior).

- This model can be solved even at finite $n$, namely without the oversimplifying thermodynamic limit $n \rightarrow \infty$.

- All the energies can be expressed in units of the thermal energy $k_{B} T \equiv \beta^{-1}$, hence, in order to avoid possible misunderstanding as $T$ already addresses the tense molecular state and to keep notation as simple as possible, in the following we set $\beta=1$, thus forcing all aforementioned parameters and variables to be dimensionless.

- As a consequence of the two previous remarks, the stochasticity is retained by the parameter $n$, such that for $n \rightarrow \infty$ stochastic computing will collapse on the deterministic one (that is, classical logic) and, on the other side, the smaller $n$ and the larger the noise affecting the system.

Now, focusing on $Z_{A}$ (as $Z_{I}$ is analogous), we define $k=\sum_{i=0}^{n} \sigma_{i}$, and we can therefore write

$$
\begin{aligned}
Z_{A} & =\sum_{(\sigma, 1)} e^{-H(\sigma, 1)}= \\
& =\sum_{k=0}^{n} A_{k} e^{-(E-k \epsilon)-h k}=e^{-E} \sum_{k=0}^{n} A_{k} e^{k(\epsilon-h)},
\end{aligned}
$$

where $A_{k}$ denotes the number of times that the sum $\sum_{i=1}^{n} \sigma_{i}$ turns out to be equal to $k$. Noting that $\sigma$ is a binary vector, we get straightforwardly that $A_{k}=\left(\begin{array}{l}n \\ k\end{array}\right)$, and therefore

$$
\begin{aligned}
Z_{A} & =e^{-E} \sum_{k=0}^{n}\left(\begin{array}{l}
n \\
k
\end{array}\right) e^{k(\epsilon-h)} \\
& =e^{-E} \sum_{k=0}^{n}\left(\begin{array}{l}
n \\
k
\end{array}\right) e^{k(\epsilon-h)} \cdot 1^{n-k} \\
& =e^{-E}\left[1+e^{(\epsilon-h)}\right]^{n} .
\end{aligned}
$$

Analogously, $Z_{I}=\left(1+e^{-h}\right)^{n}$.

Thus, the probabilities $p_{A}$ and $p_{I}$ for the complex to be in the active or in the inactive state, respectively, are

$$
\begin{aligned}
& \left(p_{A}\right)_{M M}=\frac{Z_{A}}{Z_{A}+Z_{I}}=\frac{e^{-E}\left(1+e^{\epsilon-h}\right)^{n}}{e^{-E}\left(1+e^{\epsilon-h}\right)^{n}+\left(1+e^{-h}\right)^{n}}, \\
& \left(p_{I}\right)_{M M}=\frac{Z_{I}}{Z_{A}+Z_{I}}=\frac{\left(1+e^{-h}\right)^{n}}{e^{-E}\left(1+e^{\epsilon-h}\right)^{n}+\left(1+e^{-h}\right)^{n}} .
\end{aligned}
$$

where the subscript MM stands for "Mono-Mono". 
Correspondingly,

$$
\begin{aligned}
& \left(p_{A}^{\prime}\right)_{M M}=\frac{Z_{A}}{Z_{A}+Z_{I}}=\frac{e^{E}\left(1+e^{-\epsilon-h}\right)^{n}}{e^{E}\left(1+e^{-\epsilon-h}\right)^{n}+\left(1+e^{-h}\right)^{n}}, \\
& \left(p_{I}^{\prime}\right)_{M M}=\frac{Z_{I}}{Z_{A}+Z_{I}}=\frac{\left(1+e^{-h}\right)^{n}}{e^{E}\left(1+e^{-\epsilon-h}\right)^{n}+\left(1+e^{-h}\right)^{n}} .
\end{aligned}
$$

The interesting quantity to look at is $\left(p_{A}\right)_{M M}$, as it corresponds to the concentration $\bar{T}$ of receptors in the active state and this is expected to continuously increase (respectively decrease) with the percentage of activation enhancement (i.e. $e^{-h}$, see Tab. 1) under assumptions $\mathcal{A}$ (respectively $\mathcal{A}^{\prime}$ ). We notice, though, that the original model ${ }^{39}$ is concerned with $\bar{R}$ (i.e. with $p_{I}$ ) rather than $\bar{T}$; anyhow, $p_{A}$ and $p_{I}$ carry the same information as they are complementary probabilities.

Notably, the correspondence stated in Tab. 1 confirms the consequences of assumptions (c) and (e), that is, choosing $E>0$ yields $L<1$, while choosing $\epsilon>0$ yields $c>1$. In particular, according to the notation of Ref. 39, we have

$$
\begin{gathered}
\bar{R}=\frac{(1+\alpha)^{n}}{L(1+c \alpha)^{n}+(1+\alpha)^{n},} \\
\bar{T}=1-\bar{R}=\frac{L(1+c \alpha)^{n}}{L(1+c \alpha)^{n}+(1+\alpha)^{n}} .
\end{gathered}
$$

Conclusions on the dual assumptions $\mathcal{A}^{\prime}$ are much the same and will not be repeated.

Mono-receptor/Double-ligand (MD) properties at equilibrium. Under the assumptions of the previous section, any mono-receptor/doubleligand system, built by $n$ receptors $[i \in(1,1 / 4, n)]$ and whose occupancy is ruled by $\sigma_{i}=(0,1)$, can be described by the following allosteric Hamiltonian function

$$
H(\sigma, a, I, J)=\left(E-\epsilon \sum_{i=1}^{n} \sigma_{i}\right) a+h_{1} \sum_{i \in I} \sigma_{i}+h_{2} \sum_{j \in J} \sigma_{j},
$$

where, in contrast with the previous case described by eq. (3), two distinct ligands, whose dissociation energies are denoted by $h_{1}$ and $h_{2}$ respectively, are considered. More precisely, $I$ and $J$ are two subsets of $\{1,1 / 4, n\}$ such that $I \cap J=\varnothing$, and they denote the sites linked to the first ligand and to the second ligand, respectively. To express this in formulae, we impose that $I \cup J=\left\{\right.$ indices such that $\left.\sigma_{i}=1\right\}$.

As we did for the Mono-Mono case, the partition function coupled to the Hamiltonian (18) is given by

$$
\begin{aligned}
Z & =\sum_{(\sigma, a)} e^{-H(\sigma, a)} \\
& =\sum_{(\sigma, 0)} e^{-H(\sigma, 0)}+\sum_{(\sigma, 1)} e^{-H(\sigma, 1)} \\
& =Z_{I}+Z_{A} .
\end{aligned}
$$

We focus on $Z_{A}$, as $Z_{I}$ is analogous. Let us pose $k_{1}=|I|$ and $k_{2}=|J|$, notice that $k=k_{1}+k_{2}=\sum_{i=0}^{n} \sigma_{i}$, and write the sums explicitly as

$$
Z_{A}=\sum_{(\sigma, 1)} e^{-H(\sigma, 1)}=\sum_{k=0}^{n} \sum_{k_{1}=0}^{k} A_{k, k_{1}} e^{-(E-k \epsilon)-h_{1} k_{1}-h_{2}\left(k-k_{1}\right)},
$$

where $A_{k, k_{1}}$ denotes the number of times that the sum $\sum_{i=0}^{n} \sigma_{i}$ is equal to $k$, with the condition that $k_{1}$ of the $\sigma_{i}^{\prime}$ 's belong to the set $I$. This quantity is rather tricky to calculate but can actually be rewritten in terms of multinomial coefficient (which counts the number of ways we can choose $k$ elements among $n$, with the condition that they are divided in groups of $k_{j}$ elements each). Then, we get

$$
A_{k, k_{1}}=\left(\begin{array}{c}
n \\
k_{1}, k-k_{1}
\end{array}\right)=\left(\begin{array}{c}
n \\
k_{1}, k_{2}
\end{array}\right),
$$

in such a way that $Z_{\mathrm{A}}$ can be rewritten (using $k_{1}$ and $k_{2}$ ) as

$$
\begin{aligned}
Z_{A} & =e^{-E} \sum_{k_{1}+k_{2}=0}^{n} \sum_{k_{1}=0}^{k_{1}+k_{2}}\left(\begin{array}{c}
n \\
k_{1}, k_{2}
\end{array}\right) e^{\left(k_{1}+k_{2}\right) \epsilon-h_{1} k_{1}-h_{2} k_{2}} \\
& =e^{-E} \sum_{k_{1}+k_{2}=0}^{n} \sum_{k_{1}=0}^{k_{1}+k_{2}}\left(\begin{array}{c}
n \\
k_{1}, k_{2}
\end{array}\right) e^{k_{1}\left(\epsilon-h_{1}\right)} \cdot e^{k_{2}\left(\epsilon-h_{2}\right)} \\
& =e^{-E}\left[1+e^{\left(\epsilon-h_{1}\right)}+e^{\left(\epsilon-h_{2}\right)}\right]^{n},
\end{aligned}
$$

where in the second passage we must consider a $1^{n-\left(k_{1}+k_{2}\right)}$ factor, which allows us to conclude the calculation, by simply expanding the trinomial.

Analogously, we obtain $Z_{I}=\left(1+e^{-h_{1}}+e^{-h_{2}}\right)^{n}$.

Indeed, we have

$$
\begin{aligned}
& \left(p_{A}\right)_{M D}=\frac{e^{-E}\left(1+e^{\epsilon-h_{1}}+e^{\epsilon-h_{2}}\right)^{n}}{e^{-E}\left(1+e^{\epsilon-h_{1}}+e^{\epsilon-h_{2}}\right)^{n}+\left(1+e^{-h_{1}}+e^{-h_{2}}\right)^{n}}, \\
& \left(p_{I}\right)_{M D}=\frac{\left(1+e^{-h_{1}}+e^{-h_{2}}\right)^{n}}{e^{-E}\left(1+e^{\epsilon-h_{1}}+e^{\epsilon-h_{2}}\right)^{n}+\left(1+e^{-h_{1}}+e^{-h_{2}}\right)^{n}} .
\end{aligned}
$$

In a similar fashion, under assumptions $\mathcal{A}^{\prime}$, we obtain

$$
\begin{aligned}
\left(p^{\prime}{ }_{A}\right)_{M D} & =\frac{e^{E}\left(1+e^{-\epsilon-h_{1}}+e^{-\epsilon-h_{2}}\right)^{n}}{e^{E}\left(1+e^{-\epsilon-h_{1}}+e^{-\epsilon-h_{2}}\right)^{n}+\left(1+e^{-h_{1}}+e^{-h_{2}}\right)^{n}}, \\
\left(p_{I}^{\prime}\right)_{M D} & =\frac{\left(1+e^{-h_{1}}+e^{-h_{2}}\right)^{n}}{e^{E}\left(1+e^{-\epsilon-h_{1}}+e^{-\epsilon-h_{2}}\right)^{n}+\left(1+e^{-h_{1}}+e^{-h_{2}}\right)^{n}},
\end{aligned}
$$

where the subscript $M D$ stands for "Mono-Double".

Double-receptor/Double-ligand (DD) properties at equilibrium. Under the same assumptions of the previous sections, any doublereceptor/double-ligand system, built by $n$ receptors $[i \in(1,1 / 4, n)]$ and whose occupancy is ruled by $\sigma_{i}=(0,1)$, and $\tau_{i}=(0,1)$ can be described by the following allosteric Hamiltonian function

$$
\begin{gathered}
H(\sigma, \tau, a)=H_{1}(\sigma, a)+H_{2}(\tau, a) \\
=\left(2 E-\epsilon_{1} \sum_{i=1}^{n_{1}} \sigma_{i}-\epsilon_{2} \sum_{i=1}^{n_{2}} \tau_{i}\right) a+h_{1} \sum_{i=1}^{n_{1}} \sigma_{i}+h_{2} \sum_{i=1}^{n_{2}} \tau_{i} .
\end{gathered}
$$

We note that the system factorizes into two independent MonoMono Hamiltonians, hence we can entirely skip the calculations, referring to results already presented in the section devoted to Mono-receptors/Mono-ligand properties. Thus, focusing on a symmetric case for simplicity, i.e. $\epsilon_{1}=\epsilon_{2}=\epsilon$ and $n_{1}=n_{2}=n$, we get

$\left(p_{A}\right)_{D D}=$

$\frac{e^{-2 E}\left(1+e^{\epsilon-h_{1}}+e^{\epsilon-h_{2}}+e^{\epsilon-h_{1}} e^{\epsilon-h_{2}}\right)^{n}}{\left(1+e^{-h_{1}}\right)^{n}\left(1+e^{-h_{2}}\right)^{n}+e^{-2 E}\left(1+e^{\epsilon-h_{1}}+e^{\epsilon-h_{2}}+e^{\epsilon-h_{1}} e^{\epsilon-h_{2}}\right)^{n}}$,

while, via the dual assumptions $\mathcal{A}^{\prime}$, we have

$\left(p_{A}^{\prime}\right)_{D D}=$

$\frac{e^{2 E}\left(1+e^{-\epsilon-h_{1}}+e^{-\epsilon-h_{2}}+e^{-\epsilon-h_{1}} e^{-\epsilon-h_{2}}\right)^{n}}{\left(1+e^{-h_{1}}\right)^{n}\left(1+e^{-h_{2}}\right)^{n}+e^{2 E}\left(1+e^{-\epsilon-h_{1}}+e^{-\epsilon-h_{2}}+e^{-\epsilon-h_{1}} e^{-\epsilon-h_{2}}\right)^{n}}$,

where the subscript $D D$ stands for "Double-Double". 
Logical operations. Let us now explore the possibility of using these allosteric receptor-ligand systems as operators mimicking stochastic logic gates: the presence of ligands (variables in Logic) is denoted as $S_{i}$ for the $i$-th ligand, and the presence of receptors (operators in Logic) is denoted as $R_{A, i}$ and $R_{I, i}$ for the active and inactive state of the $i$-th receptor, respectively: note that $\sigma_{i}$ and $S_{i}$ are conceptually different because, in Logic, $S_{i}$ mirrors the presence of the $i$-th ligand, that is $S_{i}=$ "true" stands for a high concentration presence of the $i$-th ligand, thus within the statistical mechanical route the $S$ 's are linked to the $h$ 's rather than to the $\sigma$ 's.

Operators are of two kinds: the unary operators YES and NOT, which evaluate a single argument, and the binary operators, e.g., AND and OR, which evaluate two arguments.

Let us describe the examples of concrete interest in the paper:

- Affirmation: "S", namely the evaluation of the presence of ligand $S$, which returns true if and only if the ligand $S$ is present. Hereafter this operator will be denoted as stochastic YES (or, in case a distinction between several ligands is necessary, as $\mathrm{YES}_{S}$ ).

- Negation: " $\neg S$ ", namely the evaluation of the absence of ligand $S$, which returns true if and only if the ligand $S$ is not present. Hereafter

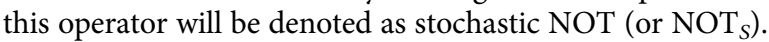

- Conjunction: " $S_{1} \wedge S_{2}$ ", namely the evaluation of the presence of both ligands, which returns "true" whenever both ligands occur to be present (i.e., in the case that $S_{1}$ and $S_{2}$ are assigned value "true") and "false" whenever at least one of the two ligands is not present (i. e., in the case that either $S_{1}$ or $S_{2}$ are assigned value false). The evaluation of such operator is hereafter denoted as $S_{1}$ AND $S_{2}$ (stochastic AND).

- Non-exclusive disjunction: $S_{1} \vee S_{2}$, namely the evaluation of the presence of at least one ligand, which returns true whenever at least one ligand is present and value false whenever they are both absent. The evaluation of such operator is hereafter denoted as $S_{1}$ OR $S_{2}$ (stochastic OR).

As we will see, the receptor molecule plays as an operator, while ligands play as variables. In order to evaluate the formula, each variable can assume value either "true" of "false" according to the ligand concentration, where "true" means that the ligand is present at a concentration larger than a threshold value, while "false" means that the ligand concentration is smaller than such a value. Moreover, the value arising from the evaluation of the operators corresponds to the activation state of the receptor: active if the evaluation returns "true" and inactive is evaluation returns "false".
Mono-receptor/Mono-ligand system: YES and NOT functions. All the plots in this and in the following sections are based on some scaling assumptions that will be discussed further in the paper (see Section "Methods"). These assumptions are essential to our purpose (that is, they enable us to tune the free variables introduced defining the Hamiltonians), and are deduced by physical and biochemical reasoning. We will refer to these assumptions as they are reported in Section Methods below.

Under scaling assumptions (35), (39) and (40), plots of the activation probability $\left(p_{A}\right)_{M M}(h)$ from eq. (12) show marked sigmoidal behavior (see Fig. 3, upper panel), signaling activation of the receptor in significative presence of the ligand, i.e. for small values of the variable $h$ : the logarithmic relation between $h$ and the concentration follows directly both from the original MWC model, as summarized in Table 1 and from the Thompson approach ${ }^{41}$.

Thus, the function $\left(p_{A}\right)_{M M}$ may be considered as mimicking the logical $\mathrm{YES}_{[L]}$ function, assuming boolean values 0 for low ligand concentration and 1 for high ligand concentration, as one can see from Tab. 2.

The threshold value is set at $\bar{h}$ which can in turn be fixed by properly choosing the system constituents (e.g. the number of binding sites hosted by a receptor).

On the contrary, the function $\left(p^{\prime}{ }_{A}\right)_{M M}$ of eq. (14) may be considered as mimicking the logical $\mathrm{NOT}_{[L]}$ function (Fig. 3, lower panel), assuming boolean values 0 for high ligand concentration and 1 for low ligand concentration, as one can see from Tab. 2.

A comparison of the theoretical versus real behavior of these gates is presented in Fig. 4, while the best fitting procedure is discussed in Section "Best fitting procedures".

Mono-receptor/Double-ligand system: $O R$ and NOR functions. The activation probability $\left(p_{A}\right)_{M D}$ (eq. (26)) can be used to model a stochastic version of the logic gate OR. In fact, if we look at the presence of the two different ligands as a binary input, the behavior of $\left(p_{A}\right)_{M D}$ (with the scaling assumptions of eqs. (35), (39)), as a function of $h_{1}$ and $h_{2}$ (see Fig. 5), recovers the OR's one (see Tab. 2). Similarly to the YES case, the value 0 for $h_{1}$ and for $h_{2}$ denotes the saturation of the ligand. Therefore, consistently with the structure of OR, the presence of only one out of the two ligands is sufficient to make the molecule active; conversely, the value $\epsilon$ denotes the absence, thus for $h_{1}=h_{2} \simeq \epsilon,\left(p_{A}\right)_{M D}$ is vanishing, namely, it returns as output "false". Note that the projection of the plot over

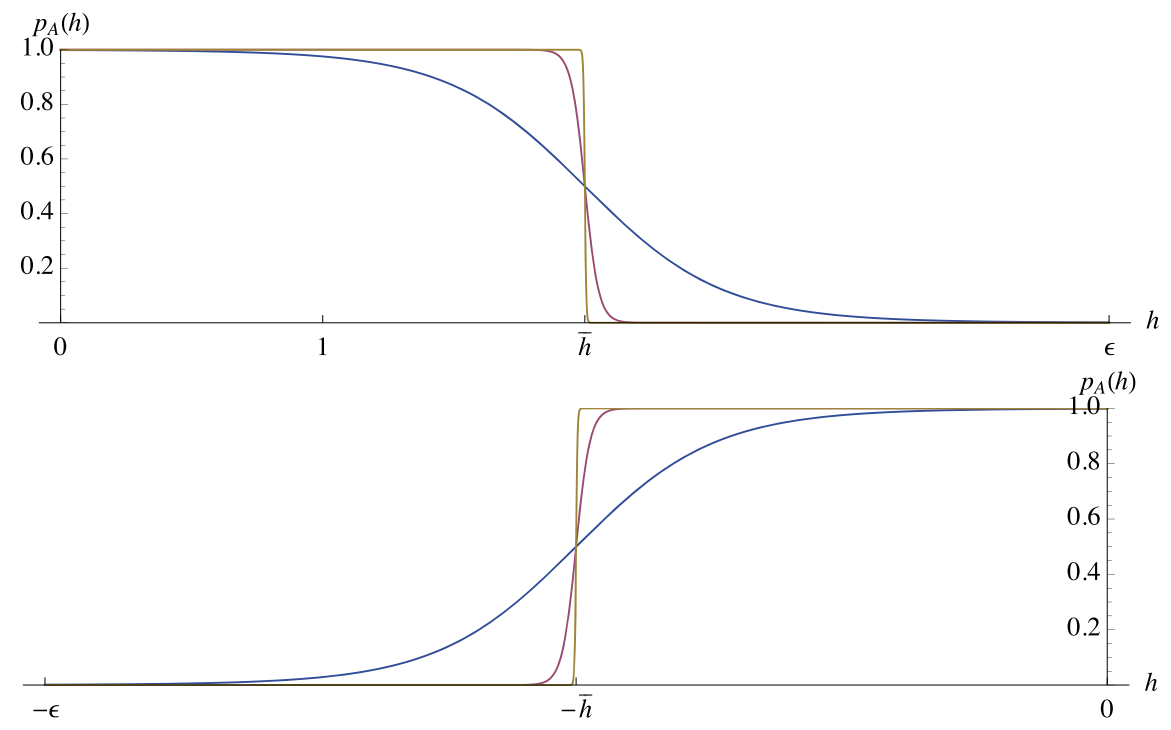

Figure 3 Upper panel: Sigmoidal behavior of $\boldsymbol{p}_{\boldsymbol{A}}(h)$ with parameters $E=\mathbf{2 n}, \epsilon=2 E / n$, where $\boldsymbol{n}=\mathbf{5}$ (blue), $\boldsymbol{n}=\mathbf{5 0}$ (red), $\boldsymbol{n}=\mathbf{5 0 0}$ (gold). Lower panel: Anti-sigmoidal behavior of $p_{A}^{\prime}(h)$ with parameters $E=2 n, \epsilon=2 E / n$, where $n=5$ (blue), $n=50$ (red), $n=500$ (gold). 
Table 2 | The truth table of all the logical operators introduced by now

\begin{tabular}{|c|c|c|c|c|c|c|c|}
\hline \multicolumn{2}{|c|}{ Input } & \multirow{2}{*}{$\frac{\mathrm{YES}_{\mathrm{A}}}{A}$} & \multirow{2}{*}{$\frac{\mathrm{NOT}_{A}}{\neg A}$} & \multirow{2}{*}{$\frac{A O R B}{A \vee B}$} & \multirow{2}{*}{$\frac{A N O R B}{A \downarrow B}$} & \multirow{2}{*}{$\frac{A \text { AND B }}{A \wedge B}$} & \multirow{2}{*}{$\frac{A N A N D B}{A \uparrow B}$} \\
\hline A & B & & & & & & \\
\hline 1 & 1 & 1 & 0 & 1 & 0 & 1 & 0 \\
\hline 1 & 0 & 1 & 0 & 1 & 0 & 0 & 1 \\
\hline 0 & 1 & 0 & 1 & 1 & 0 & 0 & 1 \\
\hline 0 & 0 & 0 & 1 & 0 & 1 & 0 & 1 \\
\hline
\end{tabular}

$h_{1}=\epsilon\left(\right.$ or $\left.h_{2}=\epsilon\right)$ gives a sigmoid, consistently with the fact that, if one of the two inputs is constantly false, the OR recovers the YES.

Performing the same calculations, the dual counterpart $\left(p_{A}^{\prime}\right)_{M D}$ of eq. (28) models the logical NOR gate, that is the direct negation of the previous one, as shown in Fig. 5).

A comparison of the theoretical versus real behavior of these gates is presented in Fig. 6 (for the OR only), whose data have been collected from an experiment sketched in Fig. 7 (lower panel); the best fitting procedure is discussed in the Section "Best fitting procedures".

Double-receptor/Double-ligand system: AND and NAND functions. The function $\left(p_{A}\right)_{D D}$ previously described (eq. (32)) models a stochastic version of the logic AND gate (see Tab. 2). As in the case of OR, we look at the two ligands as a binary input, and we assume the scaling assumptions coded in eqs. (35), (42), (44). The resulting behavior of $\left(p_{A}\right)_{D D}$ fits the one expected for the AND function, with fitness to the expected plot that sensibly improves in the extremal regions of the plot, i.e. for $h_{1,2} \sim 0, \epsilon$ (see Fig. 8). Again, its projection returns a sigmoid because if one of the two inputs is constantly true, the AND recovers the YES.

The dual version $\left(p_{A}^{\prime}\right)_{D D}$ (eq. (33)) models the logic gate NAND, i.e. the direct negation of the previous one. As this negation is precisely dual, so is the shape of the plot (see Fig. 8).

A comparison of the theoretical versus real behavior of these gates is presented in Fig. 9 (for the AND only), while, mirroring the exposition of the OR gate, Fig. 7 (upper panel) summarizes the experiment working as data source; the best fitting procedure is discussed in the Section "Best fitting procedures".

\section{Conclusions}

Chemical computing uses molecular systems to perform logical operations, mimicking processes typical of electronic devices. Advantages and disadvantages appear when comparing these two approaches to computation: chemical computing requires (for a single operation) a smaller size (Angstroms versus microns) and a lower energy consumption $\left(\sim 10^{-19}\right.$ Joule versus $\sim 10^{-9}$ Joule), yet it is slower than electronic computing (from kilohertz to megaherz versus gigahertz). Further, biochemical information processing performs at relatively large levels of noise (and this happens at different scales, ranging from enzyme-based logic gates ${ }^{10}$ to nucleic acid logic circuits $^{21}$ : noise propagates in the system as thermal disorder or in form of cross-talk among system's constituents ${ }^{4,49}$. Hence, classical (i.e. deterministic) logic works only as an ideal reference framework and the field of research would strongly benefit from a robust stochastic reformulation of logic gates whose properties can be safely tested and used as guides in in-vitro experiments.

In this work we showed that statistical mechanics can play a major role to accomplish this task: through statistical-mechanical models, we analyzed paradigmatic allosteric reaction kinetics and we proved their ability in performing noisy operations (hence working as suitable versions of stochastic logic gates). Moreover, we tested their performances (as resulting from the theory) on real data taken from enzyme-based information processing systems finding overall very good agreement between theoretical predictions and experimental behaviors. From this perspective, we showed that allosteric kinetics of mono receptor systems naturally encodes a noisy version of the input-output relations typical of the YES and NOT gates, while allosteric kinetics of double receptors systems plays as a stochastic version of the OR (NOR) and AND (NAND) gates. Finally, we checked that in the noiseless limit of the theory (for $n \rightarrow \infty$, or, alternatively, for $\beta \rightarrow \infty$ ) these gates recover the pure logic-like behavior (i.e. deterministic information processing).

The framework developed here allows establishing controllable, explicit relations among the system parameters, with remarkable practical outcomes. For instance, the noise that generally affects biological gates can be quantified permitting the management of large devices where different gates work in cascade and where noise-driven errors (possibly amplified by the various gates) actually constitute severe limitations. Also, in order to build large biochemical circuits, understanding how simple building blocks behave and how their interactions scale up with system' size is mandatory as well as quantifying the computational power of the system itself: classical reaction equations (i.e. systems of differential equations) can not accomplish these tasks, while statistical mechanics can. In fact, combining Hamiltonians coding for various gates in a cascade fashion is straightforward in the present formalization, further, an arsenal of
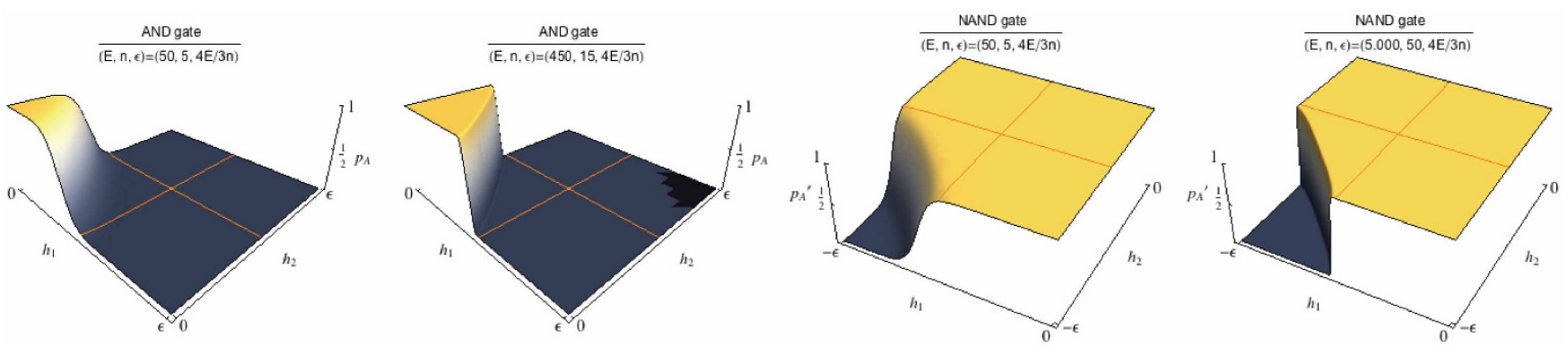

Figure $4 \mid$ Left: $\left(\boldsymbol{p}_{A}\right)_{D D}\left(\boldsymbol{h}_{1}, \boldsymbol{h}_{2}\right)$ plots. Activation of the receptor is verified by small values of $h_{1}$ and $h_{2}$, corresponding to a significative presence of both the two ligands, thus simulating a stochastic AND function. Right: $\left(p_{A}^{\prime}\right)_{M D}\left(h_{1}, h_{2}\right)$ plots. Activation of the receptor is verified by high (i.e. small in absolute value) values of $h_{1}$ or $h_{2}$, corresponding to a significative presence of any of the two ligands, thus simulating a stochastic NAND function. 


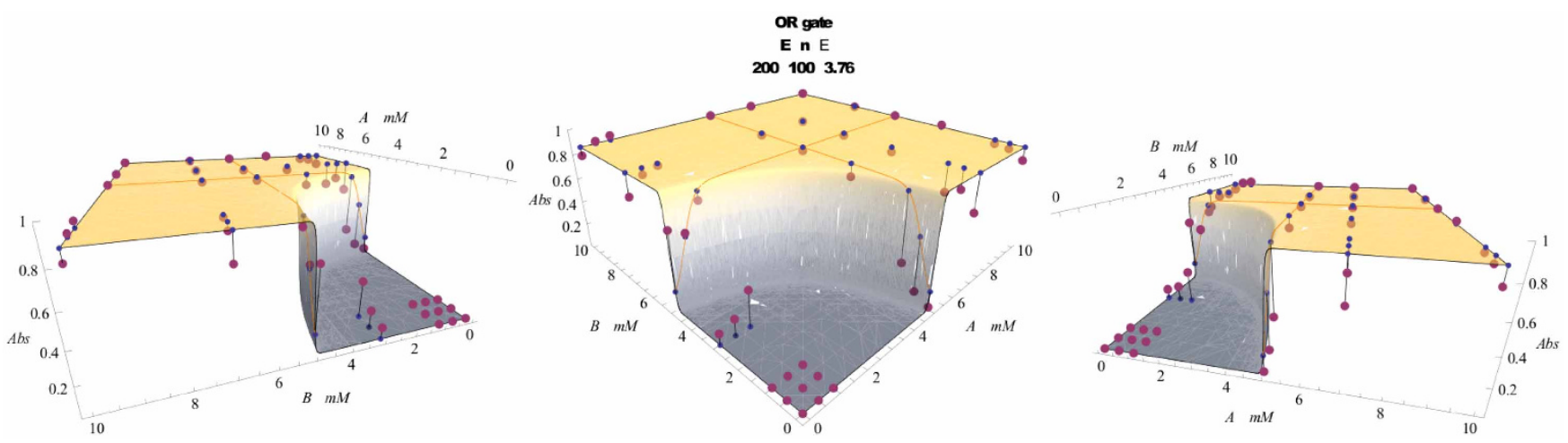

Figure $5 \mid$ The stochastic OR gate has been realized in two coupled steps involving enzymatic processes as sketched in Fig. 7: first enzyme is esterase, that reacts with ethyl butyrate ([A]) or methyl butyrate ([B]), or both, catalyzing production of ethanol and methanol, respectively. Butyric acid is a byproduct of the process. To set the gate, the physical zeros of the signals have been fixed experimentally to convenient input values (ethyl butyrate $10 \mathrm{mM}$ and methyl butyrate $10 \mathrm{mM}$ ). Bullets represent experimental data ${ }^{8}$, whereas the surface represents the best fitting according to eq. (26).

tools stemmed from the neural network branch (e.g., methods to address system's computational capacity, memory storage, etc. ${ }^{27}$ ) becomes immediately handily via this route.

\section{Methods}

In this section we discuss three major aspects of our work: the scaling assumptions, the role of allosteric cooperativity within the model and the best fitting procedures.

Scaling assumptions. As the assumption sets $\mathcal{A}$ and $\mathcal{A}^{\prime}$ only affect the sign of the parameters $E, \epsilon$ and of the variable $h$, we cannot expect every choice of these quantities to yield a realistic behavior from a biophysical viewpoint. Particularly an effective range of the variable $h$ as well as some reasonable scaling properties for $E$ and $\epsilon$ are to be determined, most likely depending on $n$.

The first issue can be solved independently of the case considered ( $M M, M D, D D)$ As evidenced in Tab. 1, for assumptions $\mathcal{A}$ it is $e^{-h}=[S] / K_{I}$ and, being $h$ positive, activation enhancement $[S] / K_{I}$ is dimensionless and ranging in $[0,1]$, thus, it may be considered as a percent molar concentration of the ligand $S$. Also, we expect that there exists a numerical (percent) value for the ligand concentration, below which the receptor activity is unaffected (see e.g. Ref. 46). We refer to this threshold value as $\tau$ and, according to Tab. 1, this also determines the significance range of $h$ as

$$
0<h<-\log \tau,
$$

which reliably limits the range of the dissociation energy to finite values. As $\tau$ determines the receptor sensitivity with respect to its activity, it is reasonably expected that $\tau \approx K_{A} / K_{I}$; in fact such inverse proportional dependence of $\tau$ with respect to $K_{I}$ is consistent with increasing monotonicity of $h$ with respect to $K_{I}$ (consistently with assumptions (c), (e)).

Moreover, from Tab. $1, \tau \approx e^{-\epsilon}$, whence a reliable significance range for $h$ is

$$
0<h<\epsilon
$$

Dually, for assumptions $\mathcal{A}^{\prime}$ it is $e^{h}=K_{I} /[S]$ and the same conclusion follows that $K_{I} /[S]$ may be considered as a percent molar concentration of the ligand $S$. As for $\tau^{\prime}$ we have $\tau^{\prime} \approx K_{I} / K_{A}$ (following from assumptions $\left(c^{\prime}\right)$, $\left(e^{\prime}\right)$ ), yielding
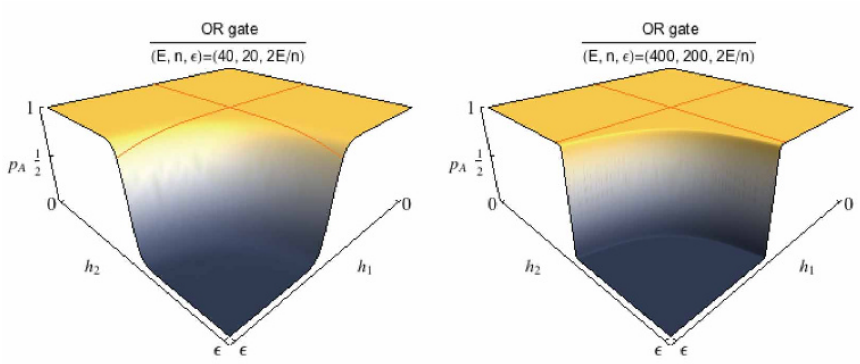

$$
-\epsilon<h<0 \text {. }
$$

Now we focus on the scaling of $E$ and $\epsilon$ : in the following we address this matter separately for the case of one or two receptors, which have different nature.

Mono-receptor case: YES/NOT and OR/NOR gates. We refer only to assumptions $\mathcal{A}$, since dual gates clearly scale in the same way. Let us start considering the Mono-Mono case: given Eq. 12, we can define $\bar{h}$ as the value of the dissociation energy such that $\left(p_{A}\right)_{M M}(\bar{h})=1 / 2$, which implies

$$
e^{-E}\left(1+e^{\epsilon-\bar{h}}\right)^{n}=\left(1+e^{-\bar{h}}\right)^{n}
$$

On the other hand, the active $(a=1)$ and saturated $(\sigma=1)$ state is an extremal state of the system corresponding to minimum entropy. As a result

$$
H(a=1, \sigma=1, \bar{h})=E-n \epsilon+\bar{h} n=0 .
$$

From the previous two equations we have

$$
\bar{h}=\frac{E}{n}, \quad \epsilon=\frac{2 E}{n},
$$

The same conclusion can be drawn independently following another route: according to the constraint (35), the maximum value attainable by the Hamiltonian (3) is $E$ and it corresponds to an active state with $h=\epsilon$; on the other hand, the minimum value attainable is $E-n \epsilon$, corresponding to $h=0$ and a fully occupied state Imposing the range interval for the energy $[E-n \epsilon, E]$ to be symmetric around 0 it must then be $E-n \epsilon=-E$, namely $\epsilon=2 E / n$. Finally we observe that $E$ depends only on the receptor, therefore in the presence of a single receptor-type it must be $E \propto n$ in view of the linear extensivity of thermodynamics; direct verification shows that

$$
E \approx 2 n
$$

best fits our purpose.
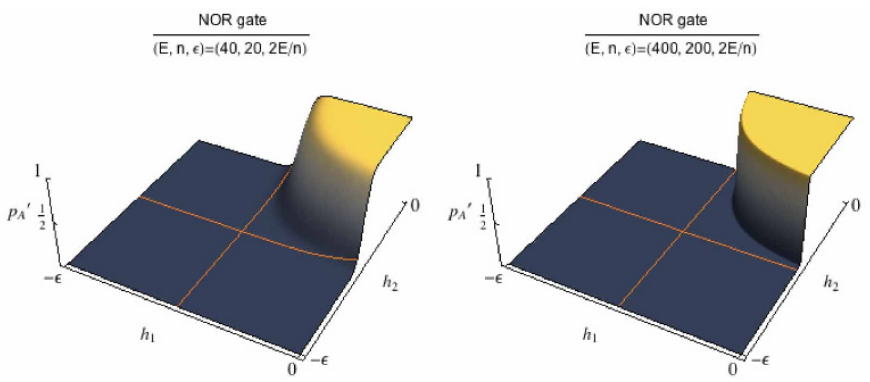

Figure 6 Left: $\left(p_{A}\right)_{M D}\left(h_{1}, h_{2}\right)$ plots. Activation of the receptor is achieved by small values of $h_{1}$ or $h_{2}$, corresponding to a significative presence of any of the two ligands, thus simulating a stochastic OR function. Right: $\left(p_{A}^{\prime}\right)_{M D}\left(h_{1}, h_{2}\right)$ plots. Activation of the receptor is verified by small values of $h_{1}$ or $h_{2}$, corresponding to a significative presence of any of the two ligands, thus simulating a stochastic NOR function. Note that for smaller $n$ curves are smooth (noisy), while for large $n$ quasi-discontinuous jumps appear.. 


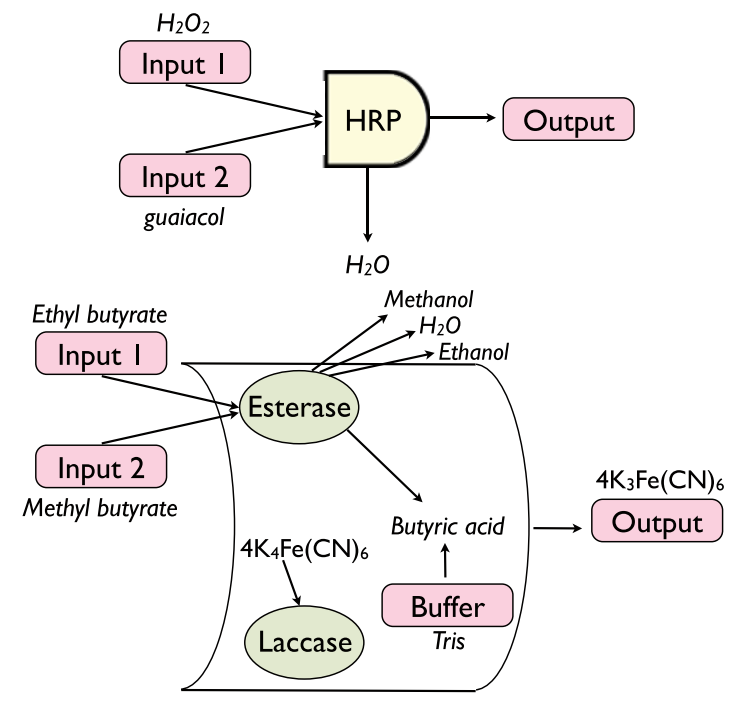

Figure 7 Schematic representation of the gates from a biochemical perspective. Upper panel: The stochastic AND gate is shown as a biocatalytic process. The two inputs are $\mathrm{H}_{2} \mathrm{O}_{2}$ and one out of three chromogens (ABTS, ferrocyanide, guaiacol) -only the latter is illustrated-. Signal processing is biocatalyzed by HRP and the output measure optically as the amount of the oxidized chromogen. See Ref. 9 for more details. Lower panel: The stochastic OR gate is shown. It involves two enzymatic processes and a buffering part. The first enzyme is esterase, that reacts with ethyl butyrate or methyl butyrate (or both) biocatalyzing production of ethanol and methanol, respectively. Butyric acid is a byproduct of the process and, as its production lowers the $p H$ of the system, further a buffer is added. The product of the process is measured by absorbance at $\lambda=$ $420 \mathrm{~nm}$ using a UV-2401PC/2501PC UV-visible spectrophotometer with a TCC-240A temperature controller holder. See Ref. 8 for more details.

Scaling assumptions for the OR gate are derived imposing that the behavior of the function $\left(p_{A}\right)_{M D}$ recovers that of $\left(p_{A}\right)_{M M}$ when one of the ligands is absent (that is, when $h_{2} \rightarrow \infty$ ). If we carry out the calculation, we find that

$$
\left.\left(p_{A}\right)_{M D}\right|_{h_{2}=\infty}=\left(p_{A}\right)_{M M},
$$

so the scaling for $E$ and $\epsilon$ must be the same of the previous one in order to be consistent.

Double-receptor case: AND/NAND gates. This case differs from the Mono-receptor one mostly because of the non-linear scaling of $E$ : since the receptors are dimeric, their response must be linear with respect to each functional monomer; consequently $E \propto n^{2}$, and again we see directly that the proper scaling is achieved by

$$
E \approx n^{2} .
$$
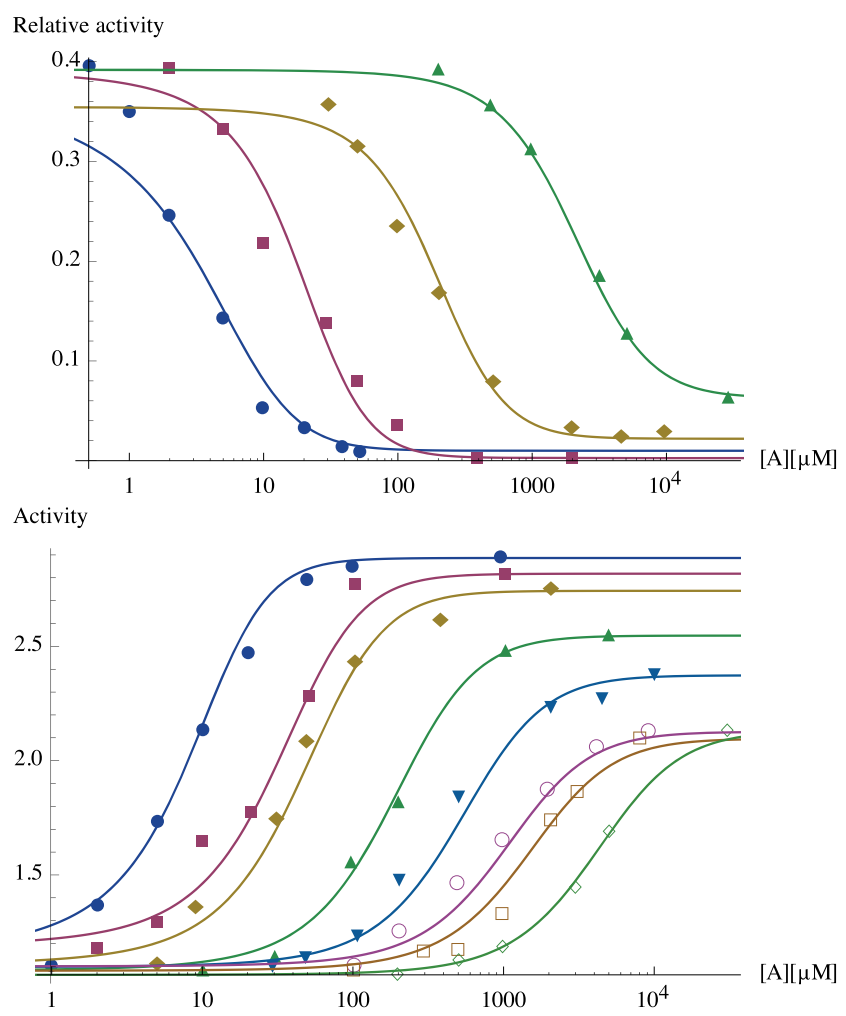

Figure 8 | Upper panel: stochastic YES gate, achieved through the statistical mechanical formulation of the allosteric monoreceptormonoligand complex under assumptions $\mathcal{A}$ and tested on $E$. colii chemotaxis network response measured by fluorescence resonance energy transfer (FRET) to decreasing concentrations (in mM) of $\alpha$ methylaspartate (MeAsp, [A]); data from Ref. 44. Lower panel: stochastic NOT gate, achieved under assumptions $\mathcal{A}^{\prime}$ and tested on E. colii FRETmeasured chemotaxis network response to increasing concentrations (in $\mathrm{mM}$ ) of MeAsp ([A]); data from Ref. 45. See Refs. 44, 45 for more details.

As far as the scaling of $\epsilon$ is concerned, we proceed in the same way as we did for the OR gate, and argue that posing $h_{2}=0$ (strong presence of one ligand) must logically recover the behavior of $\left(p_{A}\right)_{M M}$ from $\left(p_{A}\right)_{D D}$. In this case, however, we do not find an exact identity, but we can rearrange the result to look like

$$
\left.\left(p_{A}\right)_{D D}\right|_{h_{2}=0}=\frac{\frac{e^{-2 E}\left(1+e^{\epsilon}\right)^{n}}{2^{n}}\left(1+e^{\epsilon-h_{1}}\right)^{n}}{\left(1+e^{-h_{1}}\right)^{n}+\frac{e^{-2 E}\left(1+e^{\epsilon}\right)^{n}}{2^{n}}\left(1+e^{\epsilon-h_{1}}\right)^{n}},
$$

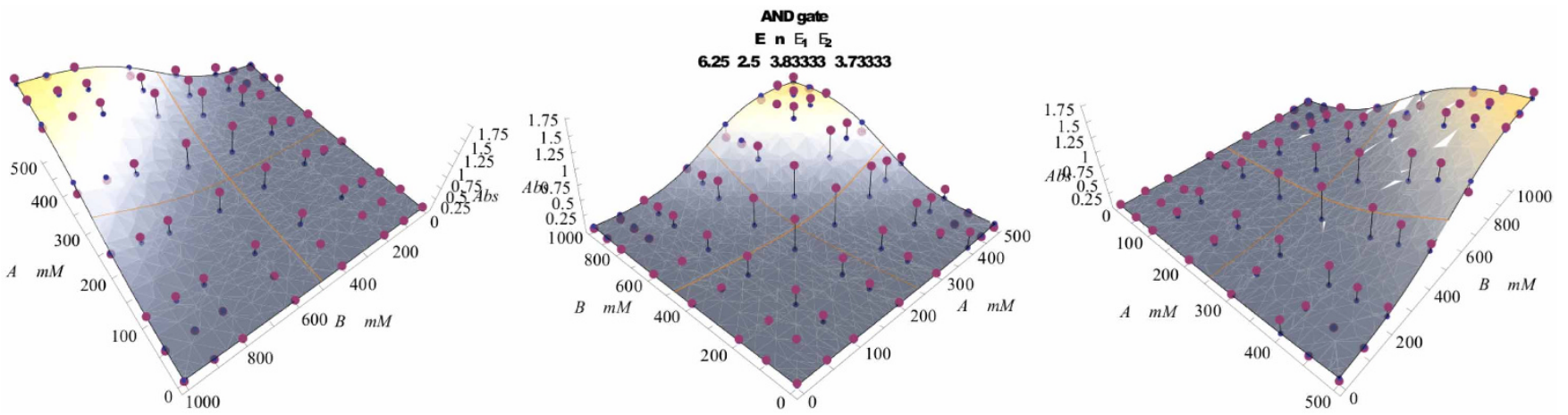

Figure 9 The stochastic AND gate has been realized by two inputs constituted by $\mathrm{H}_{2} \mathrm{O}_{2}([\mathrm{~A}])$ and guaiacol ([B]) as the chromogen, while $\mathrm{L}$-ascorbic acid $[\mathrm{Asc}](0)=120 \mu \mathrm{M}$ was used for filtering; the signal processing was biocatalyzed by HRP, as sketched in Figure 7 (upper panel). The output was measured optically as the amount of the oxidized chromogen. Bullets represent experimental data Ref. 9, whereas the surface represents the best fitting according to eq. (32). 
so, setting $e^{-E^{\text {eff }}}=\frac{e^{-2 E}\left(1+e^{\epsilon}\right)^{n}}{2^{n}}$ and $\epsilon=2 E^{\text {eff }} / n$ we obtain

$$
\epsilon \sim 4 E / 3 n
$$

The role of allosteric cooperativity. Now we want to deepen where the differences between the classical cooperativity and the MWC-like one, known in the Literature as allosteric cooperativity (see e.g., Refs. 38, 47), reside. This difference can be investigated directly from a mathematical and logical point of view by comparing the plots of the AND gate and of the OR gate.

OR gate: classical cooperativity. Here we discuss why and how the OR gate, that can be handled by a one-body statistical mechanical Hamiltonian (eq. (18)), does manifest a (roughly standard) cooperative behavior. The OR Hamiltonian is indeed a rigged one-body expression: cooperativity (meant as produced by a term $\sim J \sigma \sigma$, see eq. (45)) is nested within the definition of the OR Hamiltonian coded in eq. (18), hidden inside the request $I \cap J=\varnothing$. It is in fact possible to infer from this constraint that, in order to obtain the correct ensemble $K$ of the indices of the occupied binding sites, it is alternatively possible to introduce two subsets $I^{\prime}$ and $J^{\prime}$ where only the condition $I^{\prime}, J^{\prime} \subset N$ is left to be respected: the price to pay for this simplification, however, is in writing the ensemble $K$ as $K=I^{\prime} \sqcup J^{\prime} \backslash I^{\prime} \cap J^{\prime}$, instead of $K=I \cup J$. Such way of writing the OR constraints (which is nothing but a reformulation of the Inclusion-Exclusion Principle) makes explicit the presence of the cooperative term which turns out to be exactly $\sum_{k \in I^{\prime} \cap I^{\prime}} \sigma_{k}$. The latter can be rewritten as $\propto \sum_{i, j} I_{i j} \sigma_{i} \sigma_{j}$ (for some positive coupling $/$ ) because $\sigma_{i} \sigma_{j}=1$ if and only if both $\sigma_{i}=1$ and $\sigma_{j}=1$. As a further check of the latter statement it is to be noticed that the presence of a quadratic growth term accounting for proper cooperativity may be deduced by the circular edge of the upper plateau (Fig. 4), that is missing when looking at the AND gate (Fig. 5).

AND gate: allosteric cooperativity. In a real cooperative system there is a mutual enhancement of the activation probability; conversely, the AND gate lacks such a mutual enhancement, and the presence itself of both the ligands is simply necessary for activation, or, in other words, it is possible to (biochemically) realize an AND gate only when a (significant, that is at high concentration) amount of both ligands is present, independently of the percent concentration relative to any of them. Since the AND Hamiltonian (eq. (30)) results only from the juxtaposition of two YES Hamiltonians, it is truly one-body: this fact is fully consistent with the linear edge of the upper plateau in the AND plot (Fig. 5).

Note that, if instead of an allosteric mechanics (hence with the activation parameter $a$ and with two different conformational states $R, T$ ), we adopted a classical (i.e. notallosteric) cooperative Hamiltonian for the system, we would write

$$
\begin{aligned}
& H(\sigma, \tau)=H_{12}(\sigma, \tau)+H_{1}(\sigma)+H_{2}(\tau) \\
& =-J \sum_{i, j}^{n_{1}, n_{2}} \sigma_{i} \tau_{j}+h_{1} \sum_{i=1}^{n_{1}} \sigma_{i}+h_{2} \sum_{i=1}^{n_{2}} \tau_{i},
\end{aligned}
$$

where $J$ is a scalar parameter tuning the reciprocal enhancement.

Comparing eq. (30) and eq. (45) we see that they would be equivalent if we could write $\epsilon_{1} \equiv \epsilon_{1}(\tau)$ and $\epsilon_{2} \equiv \epsilon_{2}(\sigma)$ but, as $\log \epsilon=K_{I} / K_{A}$, then $\epsilon_{1}$ and $\epsilon_{2}$ are constant dependent on the species making up the system but independent of their bounding state, that is, $\epsilon_{1} \neq \epsilon_{1}(\tau)$ as well as $\epsilon_{2} \neq \epsilon_{2}(\sigma)$ (see Ref. 19 for classical cooperativity). Therefore, we cannot express the Hamiltonian (30) as a two-body system, and this codes for the allosteric nature of this gate.

We perform now a brief mathematical analysis of the above mentioned shape of the AND plot (from here on referred to as a "cut"): a simple calculation shows that $\left.\partial_{h_{1}}\left(p_{A}\right)_{D D}\right|_{h_{1},-h_{1}+\epsilon / 2}=\left.\partial_{h_{2}}\left(p_{A}\right)_{D D}\right|_{h_{1},-h_{1}+\epsilon / 2}$, which states that the $c u t$ is in fact corresponding to the straight line $h_{2}=-h_{1}+\epsilon / 2$ (the symmetric angular coefficient simply recalls the choice $\epsilon_{1}=\epsilon_{2}$ ). Furthermore, it is possible to prove that the slope $m$ of the line projection on the $h_{1}, h_{2}$-plane is in fact $m \approx \epsilon_{1} / \epsilon_{2}$. It follows that the case $\epsilon_{1}=\epsilon_{2}=\epsilon$ is the one best fitting the expected plot of the logical operator. On the contrary, by taking limits for either $\epsilon_{1} \rightarrow \infty$ or $\epsilon_{2} \rightarrow \infty$, one recovers the YES (respectively $\mathrm{YES}_{1}$ ) as a projection on the (orthogonal) axis.

Best fitting procedures. We can finally discuss how to test the predictions of the theory over the in vitro experiments carried on both single-input and two-inputs (bio)-logic gates: while plots summarizing our findings have already been presented, see Figures 6; 8; 9 for the reaction kinetics and Fig. 7 for a sketch of the experimental settings, here we discuss how best fits of the theory versus the data have been obtained.

Since the variable $h$ and parameters $n, E, \epsilon_{1}, \epsilon_{2}$ are dimensionless, any linear rescaling of the function $p_{A}$ is allowed (whose choice is discussed below).

Unary operators. In the YES case (data from Ref. 44), the opportune $y$-rescaling is obtained for each data set $D_{k}$ by considering the function $r_{A}^{k} \doteq\left(\max D_{k}-\min D_{k}\right) p_{A}+\min D_{k}$. In order to compensate the logarithmical progression of the axis, the $x$-rescaling (which is effectively linear, but conveyed on a $\log$ scale $)$ is of the form $r_{A}^{k}(h) \doteq\left(\max D_{k}-\min D_{k}\right) p_{A}\left(h^{m}\right)+\min D_{k}$ where $m=m_{k}$ is opportunely depending on $k$. The displayed function is $r_{A}^{\text {eff, } k}$, which is the same as $r_{A}^{k}$, but varying the parameters $n, E^{\text {eff }} \doteq 2 n+k, \epsilon^{\text {eff }} \doteq 2 E / n+\ell$. Consistently with scaling equations (39), (40), $k$ varies within $\pm 3.4 \% E$ and $\ell$ within $\pm 11.25 \% \epsilon$.
In the NOT case (data from Ref. 45) the opportune $y$-rescaling is obtained by plotting precisely the function $r_{A}^{k} \doteq\left(\max D_{k}-\min D_{k}\right) p_{A}+\min D_{k}$ with the same $x$ rescaling as in the YES case. In order to show how precise the fitting is (after suitable $\log$-lin rescaling), the best fit is obtained by considering $r_{A}^{k}$ as a function of $n=n_{k}$ only, while $E_{k}=2 n_{k}$ and $\epsilon=2 E_{k} / n_{k}$, according to the assumptions, thus the fit is practically achieved with one degree of freedom only.

We emphasize that, in both cases, the fit may be improved by data extrapolation of maximal (minimal) values for the range of $r_{A}^{k}$ which are strictly higher (lower) than the maxima (minima) of $D_{k}$.

Binary operators. Given the $x_{1} x_{2}$-data grid $\left.\left.-0,1 / 4, M_{1}\right\} \times-0,1 / 4, M_{2}\right\}$, a (vertical) $y$ rescaling is required in order to match 1 with the experimental maximum value of the activation parameter. In order to determine such value, a stable data set $S$ is opportunely defined; letting $\left\langle S_{z}\right\rangle$ be the mean $z$-value of the stable data set, we take it as a reliable value for the maximal experimental activation. The opportune $y$-rescaling is therefore obtained by considering the function $r_{A} \doteq \frac{\left\langle S_{z}\right\rangle}{p_{A}(0,0)} p_{A}$, while the $x_{1} x_{2}$ rescaling is achieved by plotting $r_{A}\left(h_{1}, h_{2}\right)=\frac{\left\langle S_{z}\right\rangle}{p_{A}(0,0)} p_{A}\left(\frac{M_{1}}{\epsilon_{1}} h_{1}, \frac{M_{2}}{\epsilon_{2}} h_{2}\right)$.

In the OR case, the stable data set is taken to be the data set in the $[8,10] \mathrm{mM} \times$ $[8,10] \mathrm{mM}$ region. The best fit is obtained by varying the parameters $n, k$ and $\ell$, where the plotted function is an effective $r_{A}$ function defined as $r_{A}^{\text {eff }}$, a function of $n$, $E^{\mathrm{eff}} \doteq 2 n+k, \epsilon^{\mathrm{eff}} \doteq \frac{E}{n}+\ell$. Consistently with scaling equations (39), (40), $k$ varies within $\pm 1 \% E$ and $\ell$ within $\pm 6.25 \% \epsilon$.

In the AND case, the stable data set is taken to be the data set in the $[400,500] \mathrm{mM}$ $\times[800,1000] \mathrm{mM}$ region. The best fit is obtained by varying parameters $n, k, \ell_{1}$ and $\ell_{2}$, where the plotted function is an effective $r_{A}$ function defined as $r_{A}^{\text {eff }}$, a function of $n$, $E^{\text {eff }} \doteq 2 n^{2}+k, \epsilon_{1,2}^{\text {eff }} \doteq \frac{2}{3} \frac{E}{n}+\ell_{1,2}$. Consistently with scaling equations (42), (44), $k$ varies within $\pm 3 \% E$ and $\ell_{1,2}$ within $\pm 15 \%$.

Results are shown in Fig. 9, for the stochastic AND, and Fig. 6 for the stochastic OR.

1. Ravasz, E., Somera, A., Mongru, D., Oltvai, Z. \& Barabasi, A. L. Hierarchical organization of modularity in metabolic networks. Science 297, 1551-1555 (2002).

2. Hartwell, L., Hopfield, J. S. L. \& Murray, A. From molecular to modular cell biology. Nature 402, C47-C52 (1999).

3. Agliari, E., Barra, A., Galluzzi, A., Guerra, F. \& Moauro, F. Multitasking associative networks. Phys. Rev. Lett. 109, 268101-268106 (2012).

4. Sollich, P., Tantari, D., Annibale, A. \& Barra, A. Extensive parallel processing or scale free networks. Phys. Rev. Lett. 113, 238106-238111 (2014).

5. Goodnow, C. Cellular and genetic mechanisms of self tolerance and autoimmunity. Nature 435, 590-597 (2005).

6. Agliari, E., Barra, A., Del Ferraro, G., Guerra, F. \& Tantari, D. Anergy in selfdirected b lymphocytes: A statistical mechanics perspective. J. Theor. Biol. DOI:10.1016/j.jtbi.2014.05.006 (2015).

7. Germain, R. The art of probable: System control in the adaptive immune system. Science 293, 240-245 (2000).

8. Zavalov, O., Bocharova, V., Privman, V. \& Katz, E. Enzyme based logic: Or gate with double sigmoid filter response. J. Phys. Chem. B 116, 9683-9692 (2012).

9. Bakshi, S., Zavalov, O., Halamek, J., Privman, V. \& Katz, E. Modularity of biochemical filtering for inducing sigmoidal response in both inputs in an enzymatic and gate. J. Phys. Chem. B 117, 9857-9872 (2013).

10. Katz, E. \& Privman, V. Enzyme-based logic systems for information processing. Chem. Soc. Rev. 39, 1835-1857 (2010).

11. Graham, I. \& Duke, T. The logical repertoire of ligand-binding proteins. Phys. Biol. 2, 159-165 (2005).

12. Prehoda, K. \& Lim, W. How signaling proteins integrate multiple inputs: a comparison of N-WASP and Cdk2. Curr. Opin. Cell Biology 14, 149-154 (2002).

13. Tamsir, A., Tabor, J. \& Voigt, C. Robust multicellular computing using genetically encoded NOR gates and chemical 'wires'. Nature 469, 212-215 (2011).

14. Kramer, B., Fischer, C. \& Fussenegger, M. BioLogic gates enable logical transcription control in mammalian cells. Biotechnol. Bioeng. 87, 478-484 (2004).

15. Setty, Y., Mayo, A., Surrette, M. \& Alon, U. Detailed map of a cis-regulatory input function. Proc. Natl. Acad. Sci. USA 100, 7702-7707 (2003).

16. Guet, C., Elowitz, M., Hsing, W. \& Leibler, S. Combinatorial synthesis of genetic networks. Science 296, 1466-1470 (2002).

17. Dueber, J., Yeh, B., Bhattacharyya, R. \& Lim, W. Rewiring cell signaling: the logic and plasticity of eukaryotic protein circuitry. Curr. Opin. Structure Biology 14, 690-699 (2004)

18. Dueber, J., Bhattacharyya, R. \& Lim, W. Reprogramming control of an allosteric signaling switch through modular recombination. Science 301, 1904-1908 (2003).

19. Agliari, E., Barra, A., Burioni, R., Di Biasio, A. \& Uguzzoni, A. Collective behaviours: from biochemical kinetics to electronic circuits. Sci. Rep. 3, 3458-3464 (2013).

20. Szacilowski, K. Infochemistry: Information Processing at the Nanoscale (Wiley, London, 2012).

21. Seeling, G., Soloveichik, D., Zhang, D. \& Winfree, E. Enzyme-free nucleic acid logic circuits. Science 314, 1585-1589 (2006). 
22. Zhang, D., Turberfield, A., Yurke, B. \& Winfree, E. Engineering entropy-driven reactions and networks catalyzed by dna. Science 318, 1121-1125 (2007).

23. Jeong, H., Tombor, B., Albert, R., Oltvai, Z. N. \& Barabsi, A. L. The large-scale organization of metabolic networks. Nature 407, 651-654 (2000).

24. Martelli, C., De Martino, A., Marinari, E., Marsili, M. \& Castillo, I. P. Identifying essential genes in escherichia coli from a metabolic optimization principle. Proc. Natl. Acad. Sc. USA 8, 2607-2611 (2009).

25. Berg, J., Lassig, M. \& Wagner, A. Structure and evolution of protein interaction networks: a statistical model for link dynamics and gene duplications. BMC Evolutionary biology 1, 51-58 (2004).

26. Boccaletti, S., Latora, V., Moreno, Y., Chavez, M. \& Hwang, D. Complex networks: Structure and dynamics. Phys. Rep. 4, 175-202 (2009).

27. Coolen, A., Kuhn, R. \& Sollich, P. Theory of neural information processing systems (Oxford University Press, Oxford, 2005).

28. Agliari, E., Annibale, A., Barra, A., Coolen, A. \& Tantari, D. Immune networks: multitasking capabilities near saturation. J. Phys. Al 41, 415003-415024 (2013).

29. Agliari, E., Barra, A., Guerra, F. \& Moauro, F. A thermodynamic perspective of immune capabilities. J. Theor. Biol. 287, 48-63 (2011).

30. Gusfield, D. Algorithms on strings, trees and sequences: computer science and computational biology (Cambridge University Press, Cambridge, 1997).

31. Ideker, T., Galitsky, T. \& Hood, L. A new approach to decoding life: Systems biology. Annu. Rev. Genomics 41, 343-372 (2001).

32. Khinchin, A. Mathematical foundations of statistical mechanics (Dover Press, New York, 1950).

33. Khinchin, A. Mathematical foundations of information theory (Dover Press, New York, 1949).

34. Jaynes, E. Information theory and statistical mechanics. part one. Phys. Rev. E 4, 620-631 (1957).

35. Jaynes, E. Information theory and statistical mechanics. part two. Phys. Rev. E 2, 171-180 (1957).

36. Von Neumann, J. The general and logical theory of automata. Cerebral mechanisms in behavior (Wiley Press, New York, 1951).

37. Chaitin, G. J. Algorithimc information theory (Wiley Press, New York, 1982).

38. Koshland, D. E., Nemethy, G. \& Filmer, D. Comparison of experimental binding data and theoretical models in proteins containing subunits. Biochemistry $\mathbf{8}$ 365-373 (1966).

39. Monod, J., Wyman, J. \& Changeaux, J.-P. On the Nature of Allosteric Transitions: A Plausible Model. J. Mol. Bio. 12, 88-118 (1965).

40. Hill, T. \& Rich, A. Cooperativity theory in biochemistry: Steady-state and equilibrium systems (Springer-Verlag New York, 1985).

41. Thompson, C. J. Mathematical Statistical Mechanics (Princeton University Press, Princeton, 1972).

42. de Ronde, W., ten Wolde, P. R. \& Mugler, A. Protein logic: A statistical mechanical study of signal integration at the single-molecule level. Biophys. J. 103, 1097-1107 (2012).
43. Mello, B. A. \& Tu, Y. An allosteric model for heterogeneous receptor complexes: Understanding bacterial chemotaxis responses to multiple stimuli. Proc. Natl. A. Sc. 102, 17354-17359 (2005).

44. Mello, B. A. \& Tu, Y. Quantitative modeling of sensitivity in bacterial chemotaxis: The role of coupling among different chemoreceptor species. Proc. Natl. A. Sc. 100, 8223-8228 (2003)

45. Keymer, J., Endres, R., Skoge, M. \& Wingreen, N. Chemosensing in escherichia coli: Two regimes of two-state receptors. Proc. Natl. Acad. Sc. USA 103, 1786-1792 (2006).

46. Linari, M., Reedy, M. K., Reedy, M. C., Lombardi, V. \& Piazzesi, G. Ca-activation and stretch-activation in insect flight muscle. Biophys. J. 87, 1101-1111 (2004).

47. Herve', G. Allosteric enzymes (CRC Press, New York, 1989).

48. Marzen, S., Garcia, H. G. \& Phillips, R. Statistical Mehcanics of Monod-WymanChangeux (MWC) Models. J. Mol. Biol. 425, 1433-1460 (2013).

49. Unger, R., Moult, J. Toward computing with Proteins Proteins: structure, function and bioinformatics 63, 53-64 (2006).

\section{Acknowledgments}

This work is supported by Gruppo Nazionale per la Fisica Matematica (INdAM) through Progetto Giovani (Agliari, 2014). A.B. is grateful to LIFE group (Laboratories for Information, Food and Energy) for partial financial support through POR Calabria FESR 2007/2013 asse 1 programma INNOVA progetto MATCH.

\section{Author contributions}

E.A. and A.B. proposed the theoretical research and gave the lines to follow. L.D.S. and M.A. made all the calculations [solving all the related problems (e.g., suitable scalings of the parameters, etc.)] and fitted the theory over the data. The latter come from the experimental route that has been completely guided by E.K. All the authors wrote the paper in a continuous synergy.

\section{Additional information}

Competing financial interests: The authors declare no competing financial interests.

How to cite this article: Agliari, E., Altavilla, M., Barra, A., Dello Schiavo, L. \& Katz, E. Notes on stochastic (bio)-logic gates: computing with allosteric cooperativity. Sci. Rep. 5, 9415; DOI:10.1038/srep09415 (2015).

This work is licensed under a Creative Commons Attribution 4.0 International License. The images or other third party material in this article are included in the article's Creative Commons license, unless indicated otherwise in the credit line; if the material is not included under the Creative Commons license, users will need to obtain permission from the license holder in order to reproduce the material. To view a copy of this license, visit http://creativecommons.org/licenses/by/4.0/ 